\title{
High-flux PGAA for milligram-weight samples
}

\author{
P. Kudejova ${ }^{1, a}$, Z. Révay ${ }^{1}$, K. Kleszcz ${ }^{1}$, C. Genreith ${ }^{2}$ and M. Rossbach ${ }^{2}$ \\ ${ }^{1}$ Heinz Maier-Leibnitz Zentrum, Technische Universität München, 85747 Garching, Germany \\ ${ }^{2}$ Institute for Energy and Climate Research, IEK-6, Forschungszentrum Jülich GmbH, 52425 Jülich, Germany
}

\begin{abstract}
With the high-intensity cold neutron flux available at the Prompt Gamma Activation Analysis (PGAA) instrument of the research reactor FRM II at the Heinz Maier-Leibnitz Zentrum (MLZ), samples with a weight of 1 $\mathrm{mg}$ or even less can be investigated for their elemental compositions using the $(\mathrm{n}, \gamma)$ capture reaction. In such cases, the typical sample packing material for PGAA experiments made of $25 \mu \mathrm{m}$ thick PTFE foil (ca. $80 \mathrm{mg}$ ) can be orders of magnitude more massive than the sample weight itself. Proper choice of the packing material and measuring conditions are then of the highest importance [1].
\end{abstract}

\section{Introduction}

The PGAA instrument started its user operation in 2008. Since then, the user group grows steadily, and more and more challenging proposals have been chosen by the referees for PGAA measurements. Having the world highest known intensity of the cold neutron flux $\left(6 \times 10^{10}\right.$ $\mathrm{cm}^{-2} \mathrm{~s}^{-1}$ thermal equivalent) implies that the accepted proposals for PGAA experiments at MLZ include a) very small samples, b) trace element analysis or c) very low capture cross-section isotopes. For these measurements, not only the highest possible neutron flux is necessary, but also the lowest possible background is essential.

Neutron beam scattered at a sample interacts with all materials in the sample vicinity and with some probability contributes to the gamma spectrum taken by a standard Compton-suppressed spectrometer (a 60\% HPGe detector coupled with a surrounding BGO scintillator) collimated by a lead shielding to see the sample position only.

To reduce the specific background in the PGAA spectra, elements intended for lining the sample chamber and sample holder are properly chosen: For neutron shielding ( ${ }^{6} \mathrm{Li}$ and ${ }^{10} \mathrm{~B}$-based) as well as for supporting the sample in the neutron beam, matrices are chosen from materials with very low neutron capture cross section like $\mathrm{C}, \mathrm{F}$-containing polymers (called Teflon, FEP or PTFE). This way, hydrogen with relatively high capture as well as scattering cross-section (333 mb and $82 \mathrm{~b}$, resp.) is exchanged by fluorine (10 $\mathrm{mb}$ and $4 \mathrm{~b}$, resp.).

\section{Experimental}

The standard measuring conditions at the PGAA instrument include a Teflon sample holder $\left(5 \times 5 \mathrm{~cm}^{2}\right)$ with PTFE fibres of $0.3 \mathrm{~mm}$ thickness holding a sample

\footnotetext{
${ }^{\mathrm{a}}$ Corresponding author: petra.kudejova@frm2.tum.de
}

usually sealed in a $25 \mu \mathrm{m}$ thick PTFE foil. Even liquid samples can be sealed that way, see Fig. 1a. Selfsupporting samples with the minimum diameter of ca. 10 $\mathrm{mm}$ like the graphite anodes of Li-Ion batteries are held by the PTFE fibres directly (Fig. 1b) to reduce unnecessary background and scattering on the PTFE bag.

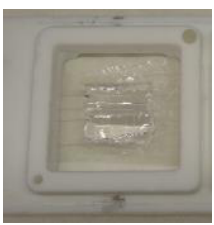

a)

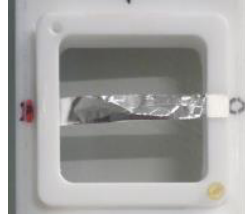

d)

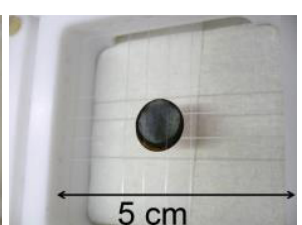

b)

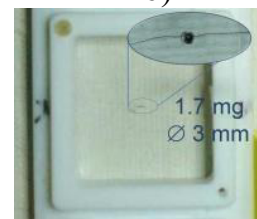

e)

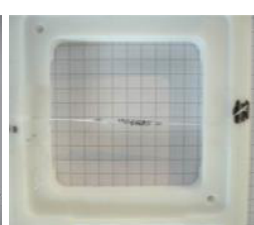

c)

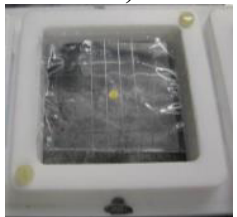

f)
Figure 1. a) Liquid PGAA sample sealed in a standard PTFE foil, b) self-holding sample of Li-ion battery anode held directly by PTFE fibres c) Micrometeorites in a 0.5- $\mu \mathrm{m}$ Mylar foil [2] d) pocket holder from $10 \mu \mathrm{m}$ thick aluminium foil e) "invisible" sample holder made of carbon fibre f) radioactive ${ }^{241} \mathrm{Am}$ sample on Au-foil, sealed between quartz plates and in a PTFE bag [4].

The 60-\% HPGe n-type detector is $35 \mathrm{~cm}$ away from the sample position, shielded heavily against scattered gamma rays and neutrons, and is collimated to the sample position by a $200 \mathrm{~mm}$ long lead collimator with a diameter of $20 \mathrm{~mm}$. A BGO annulus surrounding the detector provides the active background shielding. The data are acquired by a digital spectrometer DSPEC-50. 


\subsection{Background in the high flux}

If measured with the full flux (at the sample position $\left.4 \times 10^{10} \mathrm{~cm}^{-2} \mathrm{~s}^{-1}\right)$ in vacuum $(0.2 \mathrm{mbar})$, the background counts in the Compton-suppressed HPGe detector from the empty chamber reach 400 cps. A Teflon frame including ca. $20 \mathrm{mg}$ PTFE fibres in the direct beam adds $150 \mathrm{cps}$ and additional $80 \mathrm{mg}$ PTFE packing foil $(25 \mu \mathrm{m}$ thick) next $150 \mathrm{cps}$. For samples of ca. $1 \mathrm{mg}$ weight like the micrometeorites in Fig. 1c, a $0.5 \mu \mathrm{m}$ thick Mylar foil and a $5 \mu \mathrm{m}$ thick PTFE foil were purchased. Strongly scattering samples (like hydrogen and carbon matrix based organic samples) increase the characteristic background significantly. If carbon is to be determined in the sample, either thin aluminium (Fig. 1d) or lead foils with the thickness of less than $10 \mu \mathrm{m}$ are used to support the sample instead of Teflon. If the measurement runs in air, another $300 \mathrm{cps}$ contribute to the background spectrum increasing hydrogen signal significantly and adding nitrogen lines to it.

\subsection{Hydrogen determination}

The best background conditions for a sensitive sample measurement were reached in case of two self-holding zircaloy foils of $3 \mathrm{~mm}$ in diameter $(1.2 \mathrm{and} 1.7 \mathrm{mg})$ with a small etched hole in the middle. Here, a thin carbon fibre was used to hold the sample (Fig. 1e). Hydrogen ions were implanted in the zircaloy foil (about $190 \mathrm{ppm}$ ), and the implanted amount was to be confirmed by PGAA. Using the carbon fibre contributed with no significant counts to the background signal coming from the empty sample frame (ca. $430 \pm 20 \mathrm{cps}$ ). The neutron scattering on the sample was also reduced to its minimum by the "invisible" sample holder. That way only $0.3 \mu \mathrm{g}$ hydrogen (corresponding to $167 \pm 13 \mathrm{ppm}$ hydrogen in the sample) could be unambiguously determined. For the correct hydrogen analysis, spectrum of a similar $\mathrm{Zr}$ sample $(1.2 \mathrm{mg})$ with no implanted hydrogen was used for comparison. For the analysis, only the Zr-peak at $1405 \mathrm{keV}(0.03 \mathrm{~b})$ was used and the peak at $934 \mathrm{keV}$ $(0.125 \mathrm{~b})$ was taken for cross-checking (larger standard deviation due to more complicated spectral part and its deconvolution).

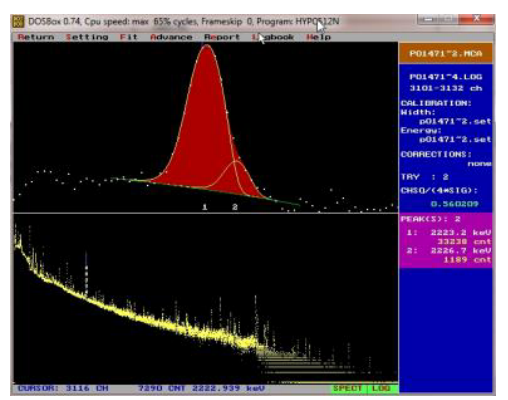

Figure 2. Significant hydrogen peak at $2223 \mathrm{keV}$ in the zircaloy sample of $1.7 \mathrm{mg}$. Fitting of the spectra is performed with Hypermet-PC [3].

\section{Radioactive samples}

In frame of the PGAA-Actinide Project, started in 2012, radioactive samples like ${ }^{237} \mathrm{~Np},{ }^{241} \mathrm{Am}$ or ${ }^{242} \mathrm{Pu}$ were measured in a cold neutron flux to evaluate the partial and total neutron capture cross-sections with a better precision than the previous literature values listed in [4]. New sample packing had to be developed to ensure low background conditions as well as secure conditions for closed radioactive sources given by the radiation safety of MLZ. In case of two ${ }^{241} \mathrm{Am}$ samples, two $0.2 \mathrm{~mm}$ thin pure Si-glass plates (Suprasil ${ }^{\circledR}$ ) glued together sandwiched the radioactive sample. Their masses were only 30 and $40 \mu \mathrm{g}$, respectively, and they were dried on a $3 \mu \mathrm{m}$ thick gold foil ( $3 \mathrm{~mm}$ in diameter) used as a neutron flux monitor (Fig. 1f). In addition, the samples were packed in PTFE foil for safety reasons. The results of the measurements are published elsewhere $[4,5]$. No vacuum could be applied and the high-intensity neutron flux was collimated to the spot of the sample with the diameter of $5 \mathrm{~mm}$ to reduce the silicon background. The samples were irradiated for $5-12 \mathrm{~h}$ and - together with prompt the decay gamma spectra taken in a low-background counting chamber next to the PGAA instrument were used for the precise data evaluation.

\section{Conclusions and Outlook}

Since increasing number of experiments at PGAA instrument of MLZ are preferably measured in "special" conditions, we are intensively searching for new sample packing and holding materials (e.g. pure carbon foils). Trace amounts of hydrogen are often a question for PGAA in a field of new material development (superconductors, fuel element cladding, etc.), so our aim is to reduce hydrogen content of shielding materials in the sample vicinity. For self-holding PGAA samples, where the non-destructivity of the method is not important and a hole for a carbon fibre can be provided, the part of the background affected by the sample itself can be reduced to its minimum. A systematic study on the mass-dependence of the spectral background is planned as well.

\section{Acknowledgement}

We acknowledge financial support by the Bundesministerium für Bildung und Forschung (BMBF) under grant $02 \mathrm{~S} 9052$.

\section{References}

1. Z. Révay, P. Kudejova, S. Söllradl, K. Kleszcz, Improvement of the PGAA facility in Garching, (in preparation for NIM-A)

2. James H. Wittke et al, Proc. Nat. Acad. Sci. 110, E2088 (2013)

3. www.iki.kfki.hu/nuclear/hypc/index_en.shtml

4. C. Genreith, M. Rossbach, Z. Revay, P. Kudejova, Nucl. Data Sheets 119, 69 (2014)

5. C. Genreith, PhD Thesis, Jülich, October 2014 